\title{
Population Structure of Phytophthora nicotianae Reveals Host-Specific Lineages on Brinjal, Ridge Gourd, and Tomato in South India
}

\author{
P. Chowdappa, B. J. Nirmal Kumar, S. P. Mohan Kumar, S. Madhura, B. Reddi Bhargavi, and M. Jyothi Lakshmi
}

First author: Director, Central Plantation Crops Research Institute, Kasaragod, Kerala; and first, second, third, fourth, fifth, and sixth authors: Indian Institute of Horticultural Research, Hesaraghatta Lake Post, Bangalore-560089, India. Accepted for publication 10 July 2016.

\begin{abstract}
Chowdappa, P., Kumar, B. J. N., Kumar, S. P. M., Madhura, S., Bhargavi, B. R., and Lakshmi, M. J. 2016. Population structure of Phytophthora nicotianae reveals host-specific lineages on brinjal, ridge gourd, and tomato in South India. Phytopathology 106:1553-1562.

Severe outbreaks of Phytophthora fruit rot on brinjal, ridge gourd, and tomato have been observed since 2011 in Andhra Pradesh, Karnataka, Telangana, and Tamil Nadu states of India. Therefore, 76 Phytophthora nicotianae isolates, recovered from brinjal (17), ridge gourd (40), and tomato (19) from different localities in these states during the June to December cropping season of 2012 and 2013, were characterized based on phenotypic and genotypic analyses and aggressiveness on brinjal, tomato, and ridge gourd. All brinjal and ridge gourd isolates were A2, while tomato isolates were both A1 (13) and A2 (6). All isolates were metalaxyl sensitive.

In addition, isolates were genotyped for three mitochondrial (ribosomal protein L5-small subunit ribosomal RNA [rpl5-rns], small subunit ribosomal RNA-cytochrome c oxidase subunit 2 [rns-cox2], and cox2+spacer) and three nuclear loci (hypothetical protein $[h y p]$, scp-like extracellular protein $[s c p]$, and beta-tubulin $[\beta-t u b])$. All regions were polymorphic but nuclear regions were more variable than mitochondrial regions. The network analysis of genotypes using the combined dataset of three nuclear regions revealed a host-specific association. However, the network generated using mitochondrial regions limited such host-specific groupings only to brinjal isolates. $P$. nicotianae isolates were highly aggressive and produced significantly $(P \leq$ $0.01)$ larger lesions on their respective host of origin than on other hosts. The results indicate significant genetic variation in the population of $P$. nicotianae, leading to identification of host-specific lineages responsible for severe outbreaks on brinjal, ridge gourd, and tomato.
\end{abstract}

The stramenopile Phytophthora nicotianae Breda de Haan (=Phytophthora parasitica Dastur) is a devastating plant pathogen on a global scale, causing foliar, fruit, root, and crown rots (Erwin and Ribiero 1996). P. nicotianae has a broad host range, including over 255 plant genera in 90 families (Mammella et al. 2013). Among the diseases caused by this pathogen, black shank of tobacco (Johnson et al. 2002) as well as gummosis and root rot of citrus species (Cacciola and Magnano di San Lio 2008) have been well studied. $P$. nicotianae is also responsible for significant losses on a number of other economically important species including fruit, oilseed, vegetable crops (Erwin and Ribiero 1996), ornamental plants, and floricultural crops (Álvarez et al. 2007; Moralejo et al. 2009; Pane et al. 2005; Prigigallo et al. 2015a, b).

The study on pathogen migration by using intraspecific genetic variability is very scanty. However, a majority of the studies to analyze intraspecific variability within $P$. nicotianae populations were limited to populations from tobacco (Hu et al. 2008; Parkunan et al. 2010; Sullivan et al. 2010; Zhang et al. 2003). Further, most of these studies were based on the random amplified polymorphic DNAs (RAPDs), which are not reliable markers for addressing the variability among populations. Although amplified fragment length polymorphism (AFLP) technique has been used to detect six clonal lineages among $P$. nicotianae isolates from nurseries of ornamental plants in Tennessee (Lamour et al. 2003), this technique has limitations for phylogenetic and genealogical analyses due to comigrating bands shared by two individuals and do not necessarily

Corresponding author: P. Chowdappa; E-mail address: pallem22@gmail.com

*The $\boldsymbol{e}$-Xtra logo stands for "electronic extra" and indicates that one supplementary table is published online.

http://dx.doi.org/10.1094/PHYTO-04-14-0099-R

(C) 2016 The American Phytopathological Society reflect descent from a common ancestor (Grünwald and Goss 2011). Recently, mitochondrial and nuclear loci containing singlenucleotide polymorphisms (SNPs) have been utilized to infer the population structure of $P$. nicotianae in relation to geographic origin and hosts (Mammella et al. 2011, 2013). Based on two polymorphic intergenic regions flanked by genes $\operatorname{trn} Y$ and $r n s$ and by genes $t r n W$ and $\operatorname{cox} 2,20$ different mitochondrial haplotypes grouped in five clades were identified in 51 isolates recovered from Italy (Mammella et al. 2011). By utilizing four mitochondrial (rpl5-rns, rns-cox2, cox2+spacer, and atp1-nad5) and three nuclear loci (hyp, scp, and $\beta$-tub) containing SNPs, 50 different multilocus mitochondrial haplotypes and 40 multilocus nuclear genotypes were identified from 96 isolates recovered from citrus, tobacco, and ornamental species in nurseries from six continents (Mammella et al. 2013).

India is the second largest producer of vegetables in world with an annual production of 87.53 million tonnes from 5.86 million hectares, accounting $14.4 \%$ of the world production. In India, potato, tomato (Solanum lycopersicum L.), chilli, cucurbits including ridge gourd (Luffa acutangula L.), brinjal (S. melongena L.), and okra are economically important and occupy approximately $50 \%$ of the total area under vegetable production. Since 2011, fruit rots caused by $P$. nicotianae have become increasingly prevalent in South India, posing a significant threat to vegetable crops like brinjal, tomato, and ridge gourd production during the southwest monsoon period (June to September). In South India, vegetable cultivation is intensive in which many crops are simultaneously and repeatedly grown. In such situations, many generations of the pathogen can take place and different genotypes or opposite mating types can come in contact thereby providing opportunity for sexual recombination and evolutionary divergence. The objectives of this study were to (i) determine the phenotypic and genotypic characteristics and aggressiveness of the $P$. nicotianae isolates collected from brinjal, ridge gourd, and tomato during a survey in 2012 and 2013, and (ii) relate these changes to the recent severe outbreaks of fruit rots on these vegetable crops in South India. 


\section{MATERIALS AND METHODS}

Sample collection and isolates of Phytophthora. Isolates were derived from naturally infected fruit samples of brinjal, ridge gourd and tomato showing typical symptoms in Bangalore rural, Chickaballapura, Hassan, and Kolar districts of Karnataka, Chittoor district of Andhra Pradesh, Hyderabad district of Telangana, Krishnagiri and Coimbatore districts of Tamil Nadu, and Thiruvanathapurm district of Kerala (Fig. 1), where severe disease epidemics occurred, during the southwest monsoon period (June to September) of 2012 and 2013. Samples consisted of three infected fruits with single lesions from each of five plants within a disease focus. Sampled fields were 10 to $15 \mathrm{~km}$ apart. From this collection, a representative set of 76 isolates were chosen that covered 76 fields in all of the important brinjal, ridge gourd, and tomato growing regions in South India (Table 1). To obtain isolates of $P$. nicotianae, fruits were disinfected by rubbing the fruit surface with a cotton pad moistened with $70 \%$ ethanol. Then, small tissue pieces $(5 \times 5 \mathrm{~mm})$ taken from the margin of the lesion were washed in sterile distilled water, surface-sterilized in $70 \%$ ethanol for $30 \mathrm{~s}$, rinsed twice in sterile distilled water, and placed on carrot agar medium (CA). After incubation at $25 \pm 1{ }^{\circ} \mathrm{C}$ under $12 \mathrm{~h}$ of fluorescent light and $12 \mathrm{~h}$ of darkness, the isolates were obtained by transferring a hyphal tip to fresh CA. Isolates were maintained on CA slants at $4{ }^{\circ} \mathrm{C}$ by periodical ( 1 to 2 months) subculturing. For long-term storage, a 5 -mm plug of actively growing mycelium from each culture was placed into a $1.5-\mathrm{ml}$ microfuge tube with $1 \mathrm{ml}$ of sterile distilled water. Isolates then were incubated for 15 days at $25^{\circ} \mathrm{C}$ before stored at $15^{\circ} \mathrm{C}$.

Morphological characterization. Isolates were grown on CA at $25 \pm 1{ }^{\circ} \mathrm{C}$ in the dark for 7 days to study colony morphology, growth rate, and production of chlamydospores. Sporangial production was stimulated by incubating three mycelia plugs $(5-\mathrm{mm}$ diameter) cut from actively growing mycelium from single hyphal tip cultures in $60 \mathrm{~mm}$ Petri dishes containing $10 \mathrm{ml}$ of sterile water extract overnight under continuous fluorescent lighting at $25 \pm 1{ }^{\circ} \mathrm{C}$. The morphological features such as sporangiophore, shape of sporangia, and the presence of papilla were recorded.

Mating type determination. The mating types of all the isolates were determined, based on the procedure described earlier (Parkunan et al. 2010), on clarified V8 juice agar by pairing with known A1 (98-75) or A2 (98-01) reference P. capsici isolates that were obtained from the Indian Institute of Spices Research, Calicut.

Metalaxyl sensitivity. Sensitivity to metalaxyl was determined by measuring radial growth of each isolate on metalaxylamended V8 juice agar (V8A) (Forbes et al. 1997). Agar plugs (5-mm-diameter) taken from a 7-day-old colony were placed on V8A amended with 5 or $100 \mu \mathrm{g}$ of metalaxyl per ml. Metalaxyl was added to V8A medium at $50^{\circ} \mathrm{C}$ just prior to dispensing it into Petri plates. Diameter of the colony of each isolate was measured after incubation in darkness at $25 \pm 1^{\circ} \mathrm{C}$ for 7 days and subtracting $5 \mathrm{~mm}$ to correct for the plug. Three replicate plates were maintained for each isolate. Each replication contained nine plates, totaling 27 Petri plates. Growth of the same isolates on V8A plates without metalaxyl served as controls. Mean percentage growth of each isolate on metalaxyl-amended substrate compared with growth on unamended substrate was calculated using previously described criteria (Forbes et al. 1997).

Aggressiveness. Zoospore suspensions of all the Phytophthora isolates were prepared separately. Sporangial production was induced by incubating overnight $10 \operatorname{discs}(3 \mathrm{~cm} \times 3 \mathrm{~cm})$ of 7-day-old mycelial CA culture in $60 \mathrm{~mm}$ Petri plates containing $10 \mathrm{ml}$ of

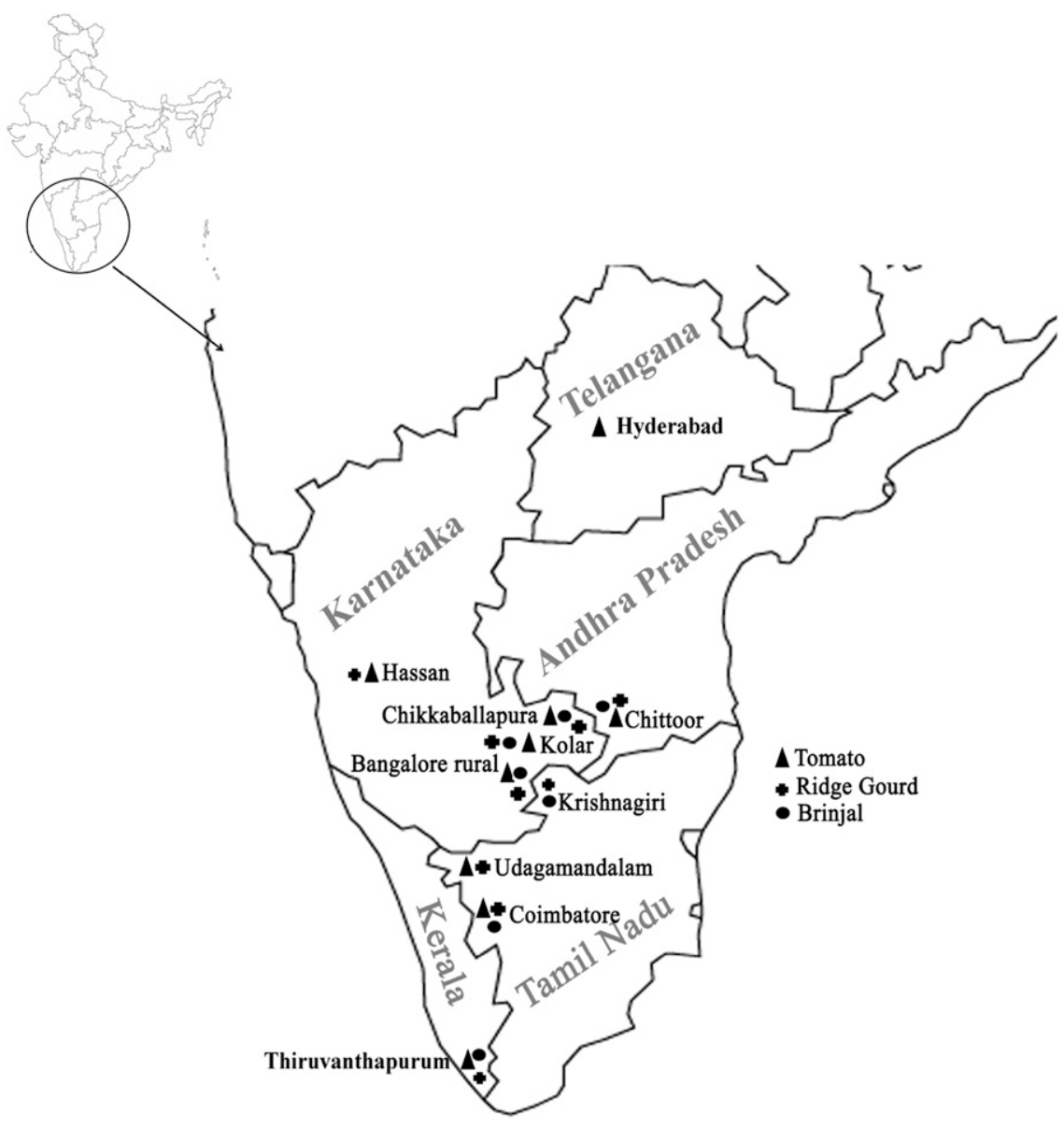

Fig. 1. Map of India where isolates of Phytophthora nicotianae from tomato, brinjal, and ridge gourd were collected. 
sterile soil water extract (Kong et al. 2003) at $25 \pm 1^{\circ} \mathrm{C}$. Zoospore release was triggered by incubating at $4^{\circ} \mathrm{C}$ for $30 \mathrm{~min}$. Zoospore suspensions were adjusted to 3,600 zoospores $/ \mathrm{ml}$ using a hemocytometer for inoculation experiments. Nearly mature green fruits of tomato cultivar Arka vikas, brinjal cultivar Arka kusmukar, and ridge gourd cultivar Arka sujata were disinfected by $1 \%$ (vol/vol) sodium hypochlorite for $1 \mathrm{~min}$ followed by three sterile distilled water rinses and air dried at room temperature $\left(25^{\circ} \mathrm{C}\right)$. Then, fruits were placed on wire mesh at $5 \mathrm{~cm}$ height in plastic chambers $(45 \mathrm{~cm}$ height $\times 40$ length $\times 15 \mathrm{~cm}$ width) containing water to maintain humidity. Fruits were inoculated with $20 \mu \mathrm{l}$ of zoospore suspension. Fruits inoculated with $20 \mu \mathrm{l}$ of sterile water served as control. Plastic chambers containing inoculated fruit samples were sealed with cellophane tape to maintain $90 \%$ humidity and incubated at $25 \pm$ $1{ }^{\circ} \mathrm{C}$ under $16 \mathrm{~h}$ photoperiod. Lesion diameters were recorded after 6 days of inoculation. Three independent experiments were conducted. Each experiment contained three replicates. Each replicate consisted of 20 fruits.

DNA extraction. Phytophthora isolates were grown in carrot broth at $25 \pm 1{ }^{\circ} \mathrm{C}$ for 7 days in dark at static conditions. Mycelia were dried on filter paper and ground in mortar with pestle using liquid nitrogen. DNA was isolated from the powdered mycelia according to the procedure described by Raeder and Broda (1985) and slightly modified by Chowdappa et al. (2003) and stored at $-20^{\circ} \mathrm{C}$ until use.
Amplification of nuclear and mitochondrial regions. Nuclear genes hyp, scp, and $\beta$-tub region were amplified with primers I11F/I12R (Schena et al. 2008), NscpF/NscpR (Mammella et al. 2013), and BtubF1/BtubR1 (Blair et al. 2008), respectively. The three mitochondrial gene regions rpl5-rns, rns-cox 2 , and cox $2+$ spacer were amplified with primers Mt13F/Mt5R (Schena and Cooke 2006), RN CoxF/RN CoxR (Mammella et al. 2013), and FM75/FM83 (Martin and Tooley 2003), respectively. PCR mixture consisted of $5 \mu \mathrm{l}$ of $10 \times$ PCR buffer $\left(15 \mathrm{mM} \mathrm{MgCl}_{2}\right), 40.75 \mu \mathrm{l}$ of sterile distilled water, $1 \mu \mathrm{l}$ of $2.0 \mathrm{mM}$ dNTPs, $1 \mu \mathrm{l}$ each of $50 \mathrm{pM}$ primers forward and reverse, $0.25 \mu \mathrm{l}$ of Taq polymerase $(5 \mathrm{U} / \mu \mathrm{l})$ (Merck Bio Sciences, India), and $1 \mu \mathrm{l}$ of genomic DNA (25 ng) for a total volume of $50 \mu \mathrm{l}$. Amplification conditions were as follows: $95^{\circ} \mathrm{C}$ for $3 \mathrm{~min}$, followed by 35 cycles $95^{\circ} \mathrm{C}$ for $1 \mathrm{~min}$, annealing $67^{\circ} \mathrm{C}$ ( hyp $), 60^{\circ} \mathrm{C}$ (scp and $\beta$-tub), $55^{\circ} \mathrm{C}$ (rpl5-rns and cox $2+$ spacer), and $64^{\circ} \mathrm{C}(r n s-\operatorname{cox} 2)$ for $1 \mathrm{~min}$, and $72^{\circ} \mathrm{C}$ for $1.5 \mathrm{~min}$, followed by a final extension of $72^{\circ} \mathrm{C}$ for $10 \mathrm{~min}$. PCR products were purified using a Nucleospin gel and PCR Clean-up (Macherey-Nagel, Germany) to remove excess primers and nucleotides. The resulting PCR product was ligated into pTZ57R/T vector and transformed into E.coli DH5 $\alpha$. For each isolate, 10 different clones were selected and extracted from the plasmids. The plasmids were sequenced in both directions with M13F and M13R primers to identify the heterozygous bases using the BigDye sequencing kit (Applied Biosystems, Foster City, CA) on

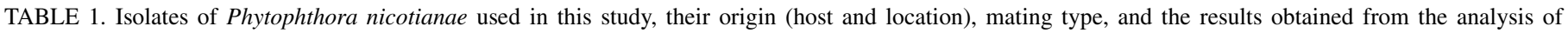
mitochondrial and nuclear $\mathrm{DNA}^{\mathrm{a}}$

\begin{tabular}{|c|c|c|c|c|c|c|c|c|c|c|c|c|c|c|}
\hline \multirow[b]{3}{*}{ Isolates } & \multirow[b]{3}{*}{ Host } & \multirow[b]{3}{*}{ Geographical origin } & \multirow[b]{3}{*}{ MT } & \multicolumn{7}{|c|}{ Mitochondrial DNA } & \multirow{2}{*}{\multicolumn{4}{|c|}{ Nuclear DNA }} \\
\hline & & & & \multicolumn{2}{|c|}{ rpl5-rns } & \multicolumn{2}{|c|}{$r n s-\operatorname{cox} 2$} & \multicolumn{2}{|c|}{$\begin{array}{l}\operatorname{cox} 2+ \\
\text { spacer }\end{array}$} & \multirow[b]{2}{*}{$\mathrm{MMH}$} & & & & \\
\hline & & & & $\overline{\mathrm{MH}}$ & $\overline{\text { Size }}$ & $\mathrm{MH}$ & $\overline{\text { Size }}$ & $\mathrm{MH}$ & $\overline{\text { Size }}$ & & hyp & $s c p$ & $\beta-t u b$ & $\overline{\mathrm{MNG}}$ \\
\hline ORgP1 & Ridge gourd & Bangalore rural, Karnataka & $\mathrm{A} 2$ & $\mathrm{H} 1$ & 769 & H1 & 567 & $\mathrm{H} 1$ & 910 & $\mathrm{H} 1$ & $\mathrm{~g} 1$ (a1) & $\mathrm{g} 1(\mathrm{a} 1)$ & g1 (a1) & G1 \\
\hline ORgP2 & Ridge gourd & Bangalore rural, Karnataka & A2 & $\mathrm{H} 1$ & 769 & $\mathrm{H} 2$ & 567 & $\mathrm{H} 1$ & 910 & $\mathrm{H} 2$ & g2 (a2) & g1 (a1) & g1 (a1) & G2 \\
\hline ORgP3 & Ridge gourd & Chickaballapura, Karnataka & A2 & $\mathrm{H} 1$ & 769 & $\mathrm{H} 2$ & 567 & $\mathrm{H} 2$ & 909 & $\mathrm{H} 3$ & g1 (a1) & g1 (a1) & g1 (a1) & G1 \\
\hline ORgP4 & Ridge gourd & Chickaballapura, Karnataka & $\mathrm{A} 2$ & H1 & 769 & $\mathrm{H} 2$ & 567 & $\mathrm{H} 1$ & 910 & $\mathrm{H} 2$ & g1 (a1) & g1 (a1) & g1 (a1) & G1 \\
\hline ORgP5 & Ridge gourd & Chickaballapura, Karnataka & $\mathrm{A} 2$ & $\mathrm{H} 1$ & 769 & H3 & 567 & $\mathrm{H} 2$ & 909 & $\mathrm{H} 4$ & g1 (a1) & g1 (a1) & g1 (a1) & G1 \\
\hline ORgP6 & Ridge gourd & Chickaballapura, Karnataka & $\mathrm{A} 2$ & $\mathrm{H} 2$ & 769 & $\mathrm{H} 2$ & 567 & $\mathrm{H} 3$ & 909 & H5 & g1 (a1) & g1 (a1) & g1 (a1) & G1 \\
\hline ORgP8 & Ridge gourd & Chickaballapura, Karnataka & $\mathrm{A} 2$ & $\mathrm{H} 1$ & 769 & $\mathrm{H} 2$ & 567 & H1 & 910 & $\mathrm{H} 2$ & g1 (a1) & g1 (a1) & g1 (a1) & G1 \\
\hline ORgP9 & Ridge gourd & Chittoor, Andhra Pradesh & A2 & $\mathrm{H} 1$ & 769 & $\mathrm{H} 2$ & 567 & $\mathrm{H} 1$ & 910 & $\mathrm{H} 2$ & g1 (a1) & $\mathrm{g} 2(\mathrm{a} 2 / \mathrm{a} 3)$ & g1 (a1) & G3 \\
\hline ORgP10 & Ridge gourd & Chittoor, Andhra Pradesh & A2 & H1 & 769 & H1 & 567 & $\mathrm{H} 1$ & 910 & $\mathrm{H} 1$ & g1 (a1) & $\mathrm{g} 2(\mathrm{a} 2 / \mathrm{a} 3)$ & g1 (a1) & G3 \\
\hline ORgP11 & Ridge gourd & Chittoor, Andhra Pradesh & $\mathrm{A} 2$ & H3 & 769 & $\mathrm{H} 2$ & 567 & $\mathrm{H} 1$ & 910 & H6 & g1 (a1) & $\mathrm{g} 1(\mathrm{a} 1)$ & g1 (a1) & G1 \\
\hline ORgP12 & Ridge gourd & Kolar, Karnataka & A2 & $\mathrm{H} 1$ & 769 & $\mathrm{H} 2$ & 567 & $\mathrm{H} 1$ & 910 & $\mathrm{H} 2$ & g1 (a1) & g1 (a1) & g1 (a1) & G1 \\
\hline ORgP13 & Ridge gourd & Kolar, Karnataka & A2 & $\mathrm{H} 1$ & 769 & $\mathrm{H} 1$ & 567 & $\mathrm{H} 1$ & 910 & $\mathrm{H} 1$ & g1 (a1) & g1 (a1) & g1 (a1) & G1 \\
\hline ORgP14 & Ridge gourd & Kolar, Karnataka & $\mathrm{A} 2$ & $\mathrm{H} 4$ & 769 & H1 & 567 & $\mathrm{H} 1$ & 910 & $\mathrm{H} 7$ & g1 (a1) & g1 (a1) & g1 (a1) & G1 \\
\hline ORgP15 & Ridge gourd & Udhagamandalam, Tamil Nadu & $\mathrm{A} 2$ & H5 & 769 & $\mathrm{H} 1$ & 567 & H1 & 910 & H8 & g3 (a3) & $\mathrm{g} 2(\mathrm{a} 2 / \mathrm{a} 3)$ & g2 (a2) & G4 \\
\hline ORgP16 & Ridge gourd & Udhagamandalam, Tamil Nadu & $\mathrm{A} 2$ & H6 & 769 & H1 & 567 & $\mathrm{H} 1$ & 910 & H9 & g1 (a1) & $\mathrm{g} 2(\mathrm{a} 2 / \mathrm{a} 3)$ & g2 (a2) & G5 \\
\hline ORgP17 & Ridge gourd & Udhagamandalam, Tamil Nadu & $\mathrm{A} 2$ & $\mathrm{H} 1$ & 769 & $\mathrm{H} 2$ & 567 & $\mathrm{H} 2$ & 909 & $\mathrm{H} 3$ & g1 (a1) & g1 (a1) & g1 (a1) & G1 \\
\hline ORgP18 & Ridge gourd & Udhagamandalam, Tamil Nadu & $\mathrm{A} 2$ & H1 & 769 & $\mathrm{H} 2$ & 567 & $\mathrm{H} 1$ & 910 & $\mathrm{H} 2$ & g1 (a1) & g1 (a1) & g1 (a1) & G1 \\
\hline ORgP19 & Ridge gourd & Udhagamandalam, Tamil Nadu & A2 & H1 & 769 & H1 & 567 & $\mathrm{H} 1$ & 910 & H1 & g1 (a1) & g3 (a4) & g1 (a1) & G6 \\
\hline ORgP20 & Ridge gourd & Udhagamandalam, Tamil Nadu & $\mathrm{A} 2$ & H3 & 769 & H1 & 567 & $\mathrm{H} 1$ & 910 & $\mathrm{H} 10$ & g1 (a1) & g1 (a1) & g3 (a3) & G7 \\
\hline ORgP21 & Ridge gourd & Udhagamandalam, Tamil Nadu & $\mathrm{A} 2$ & H5 & 769 & $\mathrm{H} 1$ & 567 & $\mathrm{H} 1$ & 910 & $\mathrm{H} 8$ & g1 (a1) & g1 (a1) & g3 (a3) & G7 \\
\hline ORgP22 & Ridge gourd & Coimbatore, Tamil Nadu & A2 & H5 & 769 & $\mathrm{H} 2$ & 567 & $\mathrm{H} 1$ & 910 & H11 & g1 (a1) & g1 (a1) & g3 (a3) & G7 \\
\hline ORgP23 & Ridge gourd & Coimbatore, Tamil Nadu & $\mathrm{A} 2$ & $\mathrm{H} 7$ & 769 & $\mathrm{H} 1$ & 567 & $\mathrm{H} 2$ & 909 & $\mathrm{H} 12$ & g1 (a1) & g3 (a4) & g1 (a1) & G9 \\
\hline ORgP25 & Ridge gourd & Coimbatore, Tamil Nadu & $\mathrm{A} 2$ & H5 & 769 & $\mathrm{H} 1$ & 567 & $\mathrm{H} 1$ & 910 & $\mathrm{H} 8$ & g1 (a1) & g1 (a1) & g3 (a3) & G7 \\
\hline ORgP26 & Ridge gourd & Coimbatore, Tamil Nadu & $\mathrm{A} 2$ & $\mathrm{H} 1$ & 769 & $\mathrm{H} 1$ & 567 & $\mathrm{H} 1$ & 910 & $\mathrm{H} 1$ & g3 (a3) & g1 (a1) & g3 (a3) & G8 \\
\hline ORgP27 & Ridge gourd & Coimbatore, Tamil Nadu & $\mathrm{A} 2$ & $\mathrm{H} 7$ & 769 & $\mathrm{H} 1$ & 567 & $\mathrm{H} 1$ & 910 & $\mathrm{H} 13$ & g1 (a1) & $\mathrm{g} 2(\mathrm{a} 2 / \mathrm{a} 3)$ & g1 (a1) & G3 \\
\hline ORgP28 & Ridge gourd & Coimbatore, Tamil Nadu & $\mathrm{A} 2$ & $\mathrm{H} 1$ & 769 & $\mathrm{H} 2$ & 567 & $\mathrm{H} 1$ & 910 & $\mathrm{H} 2$ & g1 (a1) & $\mathrm{g} 2(\mathrm{a} 2 / \mathrm{a} 3)$ & g4 (a4) & G9 \\
\hline ORgP31 & Ridge gourd & Chickaballapura, Karnataka & A2 & H1 & 769 & H1 & 567 & $\mathrm{H} 1$ & 910 & H1 & g3 (a3) & g1 (a1) & g4 (a4) & G10 \\
\hline ORgP33 & Ridge gourd & Chickaballapura, Karnataka & $\mathrm{A} 2$ & H1 & 769 & H3 & 567 & $\mathrm{H} 4$ & 908 & H14 & g4 (a4) & g1 (a1) & g5 (a5) & G11 \\
\hline ORgP34 & Ridge gourd & Bangalore rural, Karnataka & $\mathrm{A} 2$ & $\mathrm{H} 1$ & 769 & $\mathrm{H} 1$ & 567 & $\mathrm{H} 4$ & 908 & $\mathrm{H} 15$ & g5 (a5/a6) & g1 (a1) & g5 (a5) & G12 \\
\hline ORgP35 & Ridge gourd & Bangalore rural, Karnataka & $\mathrm{A} 2$ & $\mathrm{H} 1$ & 769 & $\mathrm{H} 2$ & 567 & $\mathrm{H} 4$ & 908 & H16 & g4 (a4) & g1 (a1) & g5 (a5) & G11 \\
\hline ORgP36 & Ridge gourd & Bangalore rural, Karnataka & $\mathrm{A} 2$ & $\mathrm{H} 3$ & 769 & $\mathrm{H} 2$ & 567 & $\mathrm{H} 4$ & 908 & $\mathrm{H} 17$ & g1 (a1) & g1 (a1) & g1 (a1) & G1 \\
\hline ORgP37 & Ridge gourd & Chittoor, Andhra Pradesh & $\mathrm{A} 2$ & $\mathrm{H} 1$ & 769 & $\mathrm{H} 2$ & 567 & $\mathrm{H} 4$ & 908 & H16 & g2 (a2) & g2 (a2/a3) & g1 (a1) & G13 \\
\hline ORgP39 & Ridge gourd & Kolar, Karnataka & $\mathrm{A} 2$ & $\mathrm{H} 1$ & 769 & $\mathrm{H} 1$ & 567 & $\mathrm{H} 4$ & 908 & H15 & g3 (a3) & $\mathrm{g} 1(\mathrm{a} 1)$ & g1 (a1) & G14 \\
\hline ORgP41 & Ridge gourd & Kolar, Karnataka & $\mathrm{A} 2$ & $\mathrm{H} 5$ & 769 & $\mathrm{H} 2$ & 567 & $\mathrm{H} 1$ & 910 & H11 & g2 (a2) & g1 (a1) & g1 (a1) & $\mathrm{G} 2$ \\
\hline ORgP42 & Ridge gourd & Udhagamandalam, Tamil Nadu & $\mathrm{A} 2$ & H3 & 769 & $\mathrm{H} 2$ & 567 & H1 & 910 & H6 & g1 (a1) & $\mathrm{g} 2(\mathrm{a} 2 / \mathrm{a} 3)$ & g1 (a1) & G3 \\
\hline ORgP44 & Ridge gourd & Coimbatore, Tamil Nadu & A2 & H1 & 769 & $\mathrm{H} 2$ & 567 & $\mathrm{H} 4$ & 908 & H16 & g3 (a3) & g1 (a1) & g1 (a1) & G14 \\
\hline
\end{tabular}

a MT, mating type; MH, mitochondrial haplotype; MMH, multilocus mitochondrial haplotype; and MNG, multilocus nuclear genotype. 
ABI 310 DNA Analyzer (Applied Biosystems) at Bioserve Technologies Pvt Ltd., Hyderabad.

Sequence analysis. The sequences were analyzed for reliability using their chromatograms in Bioedit 7.2.3 (Hall 1999) and consensus sequence were generated. Prior to analysis, plasmid and primers sequences were removed. Both forward and reverse sequences were analyzed for the presence of double peaks and heterozygous SNPs were identified. Heterozygous SNPs were denoted with standard degeneracy codes. Unreliable sequences were re-amplified. PCR, cloning, and sequencing process were repeated twice to confirm the SNPs and heterozygous bases.

Haplotype analysis, networks and phylogenetic analysis. Mitochondrial and nuclear genetic diversity was evaluated for each individual region and for the combined dataset of sequences. Haplotypes were identified by aligning sequences from all 76 isolates with Bioedit ver. 7.2.3 and confirmed using the DnaSP ver. 5.10.01 software (Librado and Rozas 2009). The number of polymorphic sites, haplotype or allele reconstruction, and nucleotide diversity $(\pi)$ were analyzed using DnaSP ver. 5.10.01 with gaps included in the analysis. To understand intraspecific variation, $P$. nicotianae isolates were analyzed by generating networks of isolates using a statistical parsimony algorithm implemented in TCS ver. 1.21 (Clement et al. 2000). Networks were generated for the individual nuclear (hyp, scp, and $\beta$-tub) and mitochondrial (rpl5-rns, rns-cox2, and cox2+spacer) regions.
Phylogenetic analysis was conducted for the individual and concatenated datasets of mitochondrial regions using Bayesian methods. The sequences were aligned using the multiple sequence alignment program Bioedit ver. 7.2.3. A Model test was performed to determine the best suitable model for the individual and concatenated datasets using Akaike information criterion (AIC) in Model test 3.7. As per the Model test, GTR $+\mathrm{I}+\mathrm{G}$ model with gamma distributed rate variation for all the sequence datasets was selected for the Bayesian analysis using Mr. Bayes ver. 3.2.2 (Huelsenbeck and Ronquist 2001; Ronquist and Huelsenbeck 2003). Further settings included a temperature value of 0.05 , sample frequency of 100 till the average standard deviation of split frequencies dropped below 0.01 . The $50 \%$ majority rule consensus tree was calculated where the first $25 \%$ of sampled trees were discarded as 'burn-in'. Phylogenetic trees were visualized with FigTree ver. 1.4.2 (http://tree.bio.ed. ac.uk/software/figtree/). The phylogenetic trees of all the individual and concatenated dataset were deposited in the Treebase server

To understand significant genetic structuring among originating host, combined mitochondrial haplotypes and nuclear genotypes were subjected to analysis of molecular variance (AMOVA) using Arlequin 3.1 software (Excoffier et al. 2005). Pairwise distance method was used for locus by locus analysis with 1,000 replications.

\begin{tabular}{|c|c|c|c|c|c|c|c|c|c|c|c|c|c|c|}
\hline \multirow[b]{3}{*}{ Isolates } & \multirow[b]{3}{*}{ Host } & \multirow[b]{3}{*}{ Geographical origin } & \multirow[b]{3}{*}{ MT } & \multicolumn{7}{|c|}{ Mitochondrial DNA } & \multirow{2}{*}{\multicolumn{4}{|c|}{ Nuclear DNA }} \\
\hline & & & & \multicolumn{2}{|c|}{ rpl5-rns } & \multicolumn{2}{|c|}{$r n s-\operatorname{cox} 2$} & \multicolumn{2}{|c|}{$\begin{array}{l}\text { cox } 2+ \\
\text { spacer }\end{array}$} & \multirow[b]{2}{*}{$\mathrm{MMH}$} & & & & \\
\hline & & & & MH & Size & MH & Size & MH & Size & & hyp & $s c p$ & $\beta-t u b$ & MNG \\
\hline ORgP46 & Ridge gourd & Thiruvanthapurum, Kerala & A2 & H1 & 769 & $\mathrm{H} 2$ & 567 & H5 & 909 & H18 & g3 (a3) & g3 (a4) & g1 (a1) & G15 \\
\hline ORgP47 & Ridge gourd & Hassan, Karnataka & A2 & $\mathrm{H} 3$ & 769 & $\mathrm{H} 2$ & 567 & H1 & 910 & H6 & g3 (a3) & g2 (a2/a3) & g1 (a1) & G16 \\
\hline ORgP49 & Ridge gourd & Krishnagiri, Tamil Nadu & A2 & H5 & 769 & $\mathrm{H} 2$ & 567 & H1 & 910 & H11 & g1 (a1) & g1 (a1) & g1 (a1) & G1 \\
\hline ORgP50 & Ridge gourd & Krishnagiri, Tamil Nadu & A2 & $\mathrm{H} 1$ & 769 & $\mathrm{H} 2$ & 567 & H1 & 910 & $\mathrm{H} 2$ & g1 (a1) & g2 (a2/a3) & g1 (a1) & G3 \\
\hline OBrP3 & Brinjal & Kolar, Karnataka & A2 & H9 & 769 & $\mathrm{H} 4$ & 574 & H1 & 910 & $\mathrm{H} 21$ & g6 (a7) & g4 (a5) & g6 (a6) & G17 \\
\hline OBrP4 & Brinjal & Chittoor, Andhra Pradesh & A2 & $\mathrm{H} 10$ & 769 & $\mathrm{H} 4$ & 574 & H6 & 910 & $\mathrm{H} 22$ & g6 (a7) & g4 (a5) & g6 (a6) & G17 \\
\hline OBrP5 & Brinjal & Chittoor, Andhra Pradesh & A2 & $\mathrm{H} 8$ & 769 & $\mathrm{H} 4$ & 574 & H1 & 910 & H19 & g6 (a7) & g4 (a5) & g6 (a6) & G17 \\
\hline OBrP6 & Brinjal & Chickaballapura, Karnataka & A2 & $\mathrm{H} 8$ & 769 & $\mathrm{H} 4$ & 574 & H1 & 910 & H19 & g6 (a7) & g4 (a5) & g6 (a6) & G17 \\
\hline $\mathrm{OBrP7}$ & Brinjal & Chickaballapura, Karnataka & A2 & $\mathrm{H} 8$ & 769 & $\mathrm{H} 4$ & 574 & H1 & 910 & H19 & g6 (a7) & g4 (a5) & g6 (a6) & G17 \\
\hline OBrP8 & Brinjal & Coimbatore, Tamil Nadu & A2 & $\mathrm{H} 8$ & 769 & $\mathrm{H} 4$ & 574 & H1 & 910 & H19 & g7 (a8) & g4 (a5) & g7 (a7) & G19 \\
\hline OBrP9 & Brinjal & Krishnagiri, Tamil Nadu & A2 & $\mathrm{H} 8$ & 769 & H5 & 574 & $\mathrm{H} 1$ & 910 & $\mathrm{H} 23$ & g7 (a8) & g4 (a5) & g6 (a6) & G18 \\
\hline OBrP17 & Brinjal & Chickaballapura, Karnataka & $\mathrm{A} 2$ & $\mathrm{H} 8$ & 769 & $\mathrm{H} 4$ & 574 & $\mathrm{H} 1$ & 910 & H19 & g7 (a8) & g4 (a5) & g6 (a6) & G18 \\
\hline OBrP18 & Brinjal & Chickaballapura, Karnataka & A2 & $\mathrm{H} 8$ & 769 & $\mathrm{H} 4$ & 574 & H1 & 910 & H19 & g6 (a7) & g4 (a5) & g8 (a8/a9) & G20 \\
\hline OBrP20 & Brinjal & Coimbatore, Tamil Nadu & A2 & H13 & 769 & $\mathrm{H} 4$ & 574 & $\mathrm{H} 8$ & 910 & H29 & g7 (a8) & g4 (a5) & g8 (a8/a9) & G22 \\
\hline OToP1 & Tomato & Bangalore rural, Karnataka & A2 & $\mathrm{H} 3$ & 769 & $\mathrm{H} 2$ & 567 & H1 & 910 & H6 & g1 (a1) & g1 (a1) & g9 (a10/a11) & G23 \\
\hline OToP2 & Tomato & Bangalore rural, Karnataka & $\mathrm{A} 2$ & H3 & 769 & $\mathrm{H} 2$ & 567 & H9 & 910 & $\mathrm{H} 30$ & g3 (a3) & g1 (a1) & g9 (a10/a11) & G24 \\
\hline OToP3 & Tomato & Bangalore rural, Karnataka & A1 & $\mathrm{H} 3$ & 769 & $\mathrm{H} 2$ & 567 & H9 & 910 & $\mathrm{H} 30$ & g3 (a3) & g1 (a1) & g9 (a10/a11) & G24 \\
\hline OToP5 & Tomato & Bangalore rural, Karnataka & A2 & H14 & 769 & $\mathrm{H} 2$ & 567 & H6 & 910 & $\mathrm{H} 31$ & g1 (a1/ a8) & g1 (a1) & g9 (a10) & G23 \\
\hline OToP7 & Tomato & Bangalore rural, Karnataka & A1 & H1 & 769 & $\mathrm{H} 2$ & 567 & H9 & 910 & $\mathrm{H} 32$ & g2 (a2) & g1 (a1/a6) & $\mathrm{g} 9(\mathrm{a} 11 / \mathrm{a} 12)$ & G25 \\
\hline OToP8 & Tomato & Chickaballapura, Karnataka & A1 & $\mathrm{H} 3$ & 769 & $\mathrm{H} 2$ & 567 & H6 & 910 & $\mathrm{H} 33$ & g2 (a2) & g1 (a1) & g9 (a11/a12) & G25 \\
\hline OToP9 & Tomato & Kolar, Karnataka & A1 & $\mathrm{H} 1$ & 769 & $\mathrm{H} 2$ & 567 & H6 & 910 & H34 & g3 (a3) & g2 (a2/a3) & g9 (a10/ a11) & G26 \\
\hline OToP10 & Tomato & Kolar, Karnataka & A1 & H1 & 769 & $\mathrm{H} 2$ & 567 & $\mathrm{H} 10$ & 910 & H35 & g3 (a3) & g2 (a2/a3) & g9 (a10/ a11) & G26 \\
\hline OToP11 & Tomato & Chittoor, Andhra Pradesh & A1 & $\mathrm{H} 14$ & 769 & $\mathrm{H} 2$ & 567 & $\mathrm{H} 1$ & 910 & H36 & g1 (a1) & g1 (a1) & g9 (a10/ a11) & G23 \\
\hline OToP12 & Tomato & Coimbatore, Tamil Nadu & A1 & $\mathrm{H} 1$ & 769 & $\mathrm{H} 2$ & 567 & H11 & 910 & H37 & g2 (a2) & g1 (a1/a6) & g9 (a11/a12) & G25 \\
\hline
\end{tabular}




\section{RESULTS}

Morphological characterization. All 76 isolates of Phytophthora obtained from brinjal (17), ridge gourd (40), and tomato (19) in different localities of South India matched the description of P. nicotianae based on taxonomic keys (Stamps et al. 1990) and morphological characteristics such as noncaducous and ovoid sporangia (Fig. 2). All isolates from brinjal, ridge gourd, and tomato produced colonies with no specific pattern and dense or low spreading aerial mycelium on CA. The radial growth rate on $\mathrm{CA}$ at

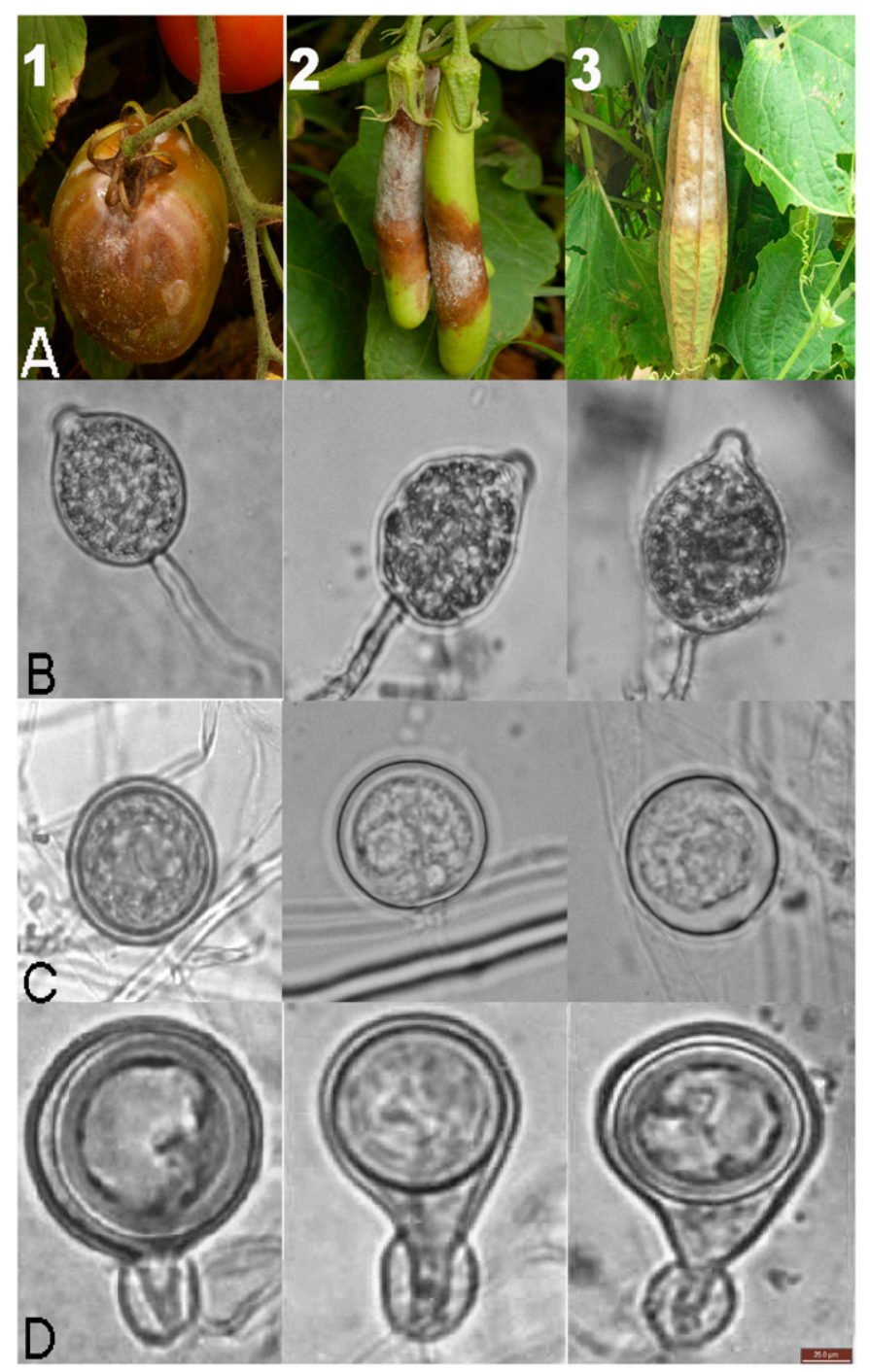

Fig. 2. Disease symptoms, sporangial, chlamydospore, and oospore morphology of Phytophthora nicotianae isolates: 1, tomato; 2, brinjal; and 3, ridge gourd. A, Disease symptoms. B, Sporangial morphology. C, Chlamydospore morphology.

D, Oospore morphology. $25^{\circ} \mathrm{C}$ varied from 6 to $8 \mathrm{~mm} /$ day. All isolates produced abundant sporangia in liquid cultures under light, rarely on CA in light or dark. Sporangia were borne singly on sympodium and noncaducous. Sporangia were predominately spherical, ovoid, obturbinate with a prominent papilla (Fig. 2). The dimensions of sporangia ranged from 26 to $53 \times 22$ to $39 \mu \mathrm{m}$ with length: breadth ratio of 1.1 to 1.3 . On CA, all isolates produced abundant spherical chlamydospores that ranged from 21 to $54 \mu \mathrm{m}$ diameter within 15 days in dark, either terminal or intercalary (Fig. 2). All isolates were heterothallic; the brinjal and ridge gourd isolates were A2 while tomato were both A1 (14) and A2 (5) mating types (Table 1). Antheridia were mostly amphigynous, cylindrical and varied from 9 to $13 \times 8$ to $10 \mu \mathrm{m}$. Oogonia varied from 15 to $32 \mu \mathrm{m}$ diameter and oospores were in the range of 13 to $28 \mu \mathrm{m}$ diameter (Fig. 2). All P. nicotianae isolates had mycelial growth less than $40 \%$ of the growth of the control at $5 \mu \mathrm{g} / \mathrm{ml}$ of metalaxyl and were considered metalaxyl sensitive.

Aggressiveness. All isolates produced typical symptoms with white mycelial growth and sporangia after 6 days of inoculation on detached fruits of tomato cultivar Arka vikas, brinjal cultivar Arka kusumakar, and ridge gourd cultivar Arka sujata. Cross inoculation tests with all isolates indicated a very clear pattern of pathogenic aggressiveness; isolates produced larger lesions on their respective host of origin than on their alternative host (Table 2). Hence,

TABLE 3. Lesion area $\left(\mathrm{cm}^{2}\right)$ on detached fruits of tomato, brinjal, and ridge gourd by isolates of Phytophthora nicotianae originated from different hosts ${ }^{\mathrm{a}}$

\begin{tabular}{|c|c|c|c|}
\hline \multirow[b]{2}{*}{ Isolates } & \multicolumn{3}{|c|}{ Lesion size $\left(\mathrm{cm}^{2}\right)$ on detached fruits } \\
\hline & Tomato & Ridge gourd & Brinjal \\
\hline \multicolumn{4}{|l|}{ Tomato } \\
\hline OToP1 & $9.44 \pm 0.21$ & $6.17 \pm 0.11$ & $6.29 \pm 0.48$ \\
\hline OToP2 & $9.52 \pm 0.84$ & $6.28 \pm 0.98$ & $6.45 \pm 0.36$ \\
\hline OToP3 & $9.92 \pm 0.18$ & $6.66 \pm 0.13$ & $6.30 \pm 0.84$ \\
\hline OToP4 & $9.66 \pm 0.98$ & $6.29 \pm 0.45$ & $6.43 \pm 0.53$ \\
\hline OToP5 & $9.28 \pm 0.87$ & $6.39 \pm 0.87$ & $6.08 \pm 0.45$ \\
\hline $\mathrm{CD} 1 \%^{\mathrm{b}}$ & 1.18 & 0.45 & 0.43 \\
\hline \multicolumn{4}{|l|}{ Brinjal } \\
\hline OBrP1 & $6.26 \pm 0.11$ & $6.13 \pm 0.63$ & $10.62 \pm 0.45$ \\
\hline $\mathrm{OBrP} 2$ & $6.32 \pm 0.86$ & $6.43 \pm 0.76$ & $10.47 \pm 0.67$ \\
\hline $\mathrm{OBrP} 3$ & $6.54 \pm 0.77$ & $7.94 \pm 0.64$ & $10.47 \pm 0.67$ \\
\hline $\mathrm{OBrP} 4$ & $6.39 \pm 0.54$ & $6.43 \pm 0.13$ & $10.86 \pm 0.56$ \\
\hline OBrP5 & $6.31 \pm 0.54$ & $6.26 \pm 0.94$ & $10.72 \pm 0.47$ \\
\hline CD1\% & 0.93 & 0.55 & 1.40 \\
\hline \multicolumn{4}{|c|}{ Ridge gourd } \\
\hline ORgP1 & $6.35 \pm 0.31$ & $10.82 \pm 0.26$ & $6.29 \pm 0.47$ \\
\hline ORgP2 & $6.43 \pm 0.35$ & $10.74 \pm 0.45$ & $6.38 \pm 0.56$ \\
\hline ORgP3 & $6.28 \pm 0.87$ & $10.46 \pm 0.45$ & $6.92 \pm 0.43$ \\
\hline ORgP4 & $6.07 \pm 0.45$ & $10.98 \pm 0.27$ & $6.09 \pm 0.38$ \\
\hline ORgP5 & $6.13 \pm 0.43$ & $10.88 \pm 0.45$ & $6.06 \pm 0.36$ \\
\hline CD1\% & 0.42 & 0.40 & 0.31 \\
\hline
\end{tabular}

a Significant differences were found between origin of isolates (tomato, brinjal, and ridge gourd) and host at $P>0.01$ according Fischer's least significant difference test. No significant differences were recorded within isolates originating from single host, so data were shown for five isolates from each host. \pm represents standard error values.

b $\mathrm{CD}$ denotes critical difference at $1 \%$ level.

TABLE 2. Summary table showing results from the analyses of mitochondrial and nuclear sequences of Phytophthora nicotianae

\begin{tabular}{|c|c|c|c|c|c|c|c|c|}
\hline Gene & rpl5-rns & $r n s-\operatorname{cox} 2$ & $\operatorname{cox} 2$-spacer & $\mathrm{CMS}^{\mathrm{a}}$ & Hyp & $s c p$ & $\beta-t u b$ & $\mathrm{CNS}^{\mathrm{a}}$ \\
\hline Isolates & 76 & 76 & 76 & 76 & 76 & 76 & 76 & 76 \\
\hline Alignment length (bp) & 769 & 574 & 910 & 2,253 & 240 & 544 & 1,038 & 1,822 \\
\hline Haplotype/allele number & 14 & 7 & 14 & 42 & 7 & 4 & 10 & 32 \\
\hline Haplotype/allele diversity & 0.7730 & 0.6540 & 0.6912 & 0.9646 & 0.756 & 0.669 & 0.816 & 0.946 \\
\hline Polymorphic sites & 14 & 40 & 14 & 68 & 4 & 5 & 28 & 37 \\
\hline Site with gaps & 0 & 7 & 3 & 10 & 0 & 0 & 0 & 0 \\
\hline Parsimony informative sites & 7 & 35 & 14 & 56 & 4 & 5 & 26 & 35 \\
\hline SNPs & 14 & 47 & 17 & 78 & 4 & 5 & 25 & 34 \\
\hline Nucleotide diversity $(\pi)$ & 0.0024 & 0.0048 & 0.0021 & 0.0029 & 0.0057 & 0.0033 & 0.0043 & 0.0042 \\
\hline
\end{tabular}

a CMS, combined mitochondrial sequences; CNS, combined nuclear sequences. 
significant differences were found between origin of isolates and host at $P \leq 0.01$ according to Fischer's least significant difference test. No significant differences were recorded within isolates originating from a single host, which was clearly evident when mean lesion diameter values were examined.

Analysis of intraspecific variation. Amplification and sequencing. Three mitochondrial (rpl5-rns, rns-cox 2 , and $\operatorname{cox} 2+$ spacer) and three nuclear ( $h y p, s c p$, and $\beta$-tub) regions were amplified and sequenced from all 76 isolates (Table 1) for determining intraspecific variation. DNA sequences were deposited in GenBank with the following accession numbers (Supplementary Table S1): KJ506466 to KJ506541 (rpl5-rns), KJ506314 to KJ506389 (rns-cox2), KJ506390 to KJ506465 (cox2+spacer), KJ506542 to KJ506617 (hyp), KJ506238 to KJ506313 (scp), and KJ506618 to
KJ506693 $(\beta$-tub). All regions were polymorphic but nuclear regions had higher nucleotide diversity than mitochondrial regions (Tables 1 and 3).

Nuclear DNA. The analyses of nuclear regions indicated the presence of intraspecific polymorphisms in the three loci examined (Tables 1 and 3). In all, 4, 5, and 25 SNPs differentiated 7 (haplotype diversity of 0.756), 4 (0.6690), and 10 genotypes (0.816) in the hyp, $s c p$, and $\beta$-tub nuclear loci, respectively. From the combined dataset of hyp, scp, and $\beta$-tub nuclear sequences $(1,822 \mathrm{bp}), 34$ SNPs were identified (average of 1 SNP for every $53 \mathrm{bp}$ ) and differentiated 32 different multilocus nuclear genotypes with high haplotype diversity (0.946) (Table 3). The number of heterozygous SNPs were higher in $\beta$-tub sequences than other nuclear regions, due to high haplotype diversity (0.816).

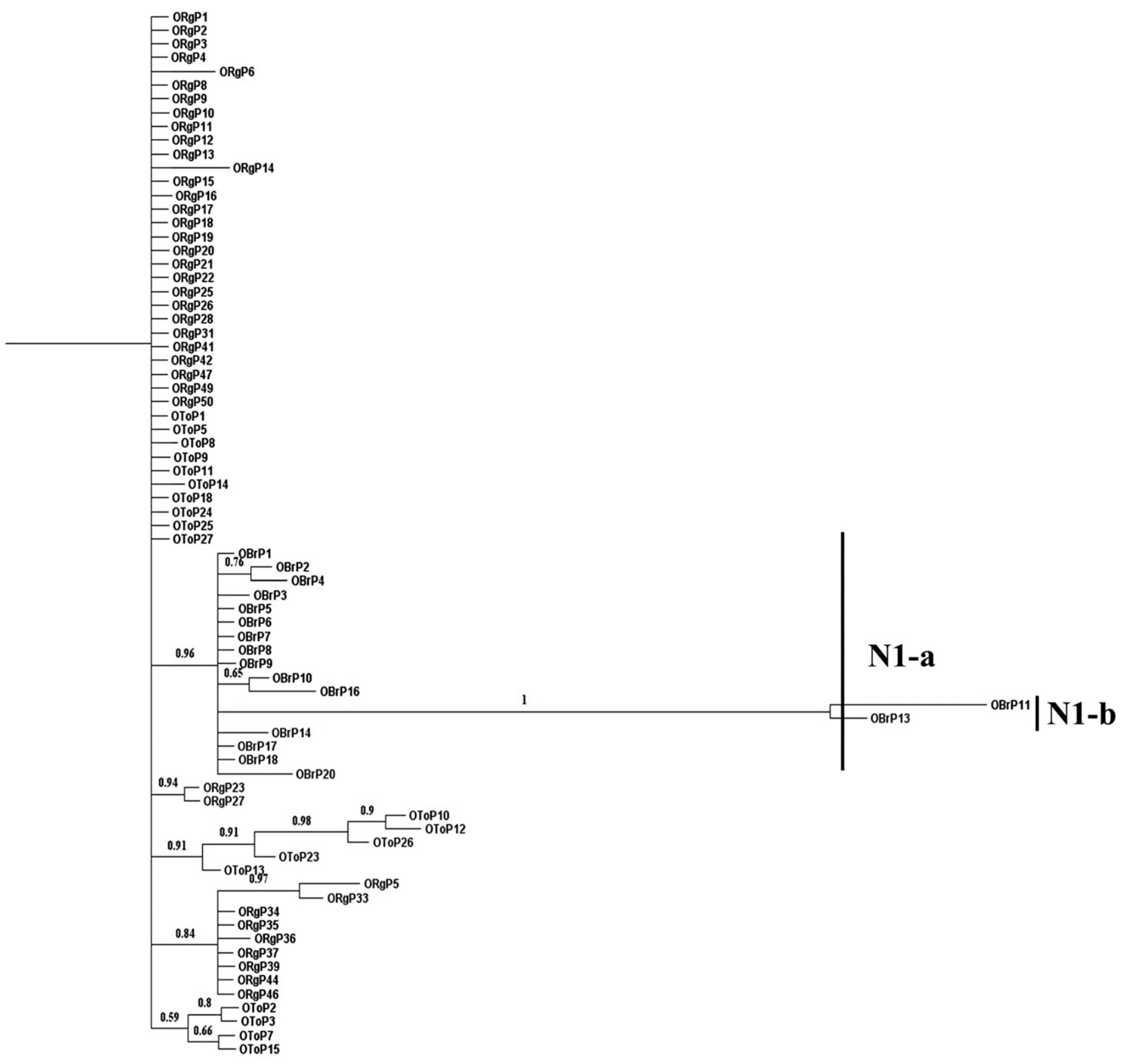

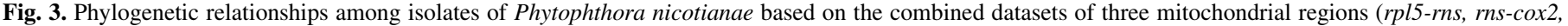
and cox $2+$ spacer). Numbers on nodes represent the posterior probability values for statistical support to Bayesian method. 
Mitochondrial DNA. Intraspecific variation was also noticed in the mitochondrial regions analyzed (Tables 1 and 3). The rpl5-rns intergenic region (769 bp) contained 14 SNPs and no gaps giving $\pi$ of 0.0024 and identified 14 mitochondrial haplotypes. The rns-cox 2 region was variable in length from 567 to $574 \mathrm{bp}$ with $\pi$ of 0.0048 (Table 3). The rns-cox 2 region consisted of 47 SNPs and seven sites with gaps and identified seven mitochondrial haplotypes. The cox2spacer region had a length between 907 and 910 bp (Table 1) and $\pi$ of 0.0021 (Table 3 ). The cox 2 -spacer region consisted of 17 SNPs and 3 sites with gaps identifying 14 mitochondrial haplotypes. Haplotype diversity for regions rpl5-rns, rns-cox2, and cox2+spacer were in the range of 0.6540 to 0.7730 (Table 3 ). The combined dataset for the three mitochondrial regions revealed 78 SNPs at an average of 1 SNP for every 29 bp and 10 sites with gaps representing 2,253 bases, which identified 42 different multilocus mitochondrial haplotypes with a high haplotype diversity (0.9646) (Table 3).

Phylogenetic analyses. Phylogenetic analyses were conducted using the combined dataset of the three mitochondrial regions. The phylogenetic tree constructed from the concatenated dataset of sequences of the three mitochondrial regions revealed that brinjal isolates formed a separate clade (N1), consisting of 11 haplotypes representing 17 isolates, that were separated into two subclades, N1-a and N1-b (Figs. 3 and 4). Phylogenetic tree constructed from the individual gene sequence of $\beta$-tub region demarcated hostspecific grouping of isolates with strong node support (posterior probabilities $>0.95$ ).

Network analysis. Forty-two haplotypes were generated using mitochondrial sequences. All 17 isolates from brinjal clustered together into 11 haplotypes (H19 to H29). There was no host wise grouping in ridge gourd and tomato isolates. The network analysis of genotypes using the combined dataset of three nuclear regions revealed a relevant association between host and multilocus genotypes (Fig. 5). All 17 isolates from brinjal representing six genotypes (G17, G18, G19, G20, G21, and G22) clustered together. Similarly, all 40 ridge gourd isolates representing 16 genotypes (G1 to G16) formed a separate group. Tomato isolates grouped in 10 genotypes (G23 to G32) formed a separate cluster. The network generated using nuclear regions revealed host-specific groups compared with mitochondrial regions.

AMOVA. AMOVA revealed a significant host-specific genetic structure with both combined nuclear genotypes and mitochondrial haplotypes, which explained 67.70 and $53.99 \%$ of the total

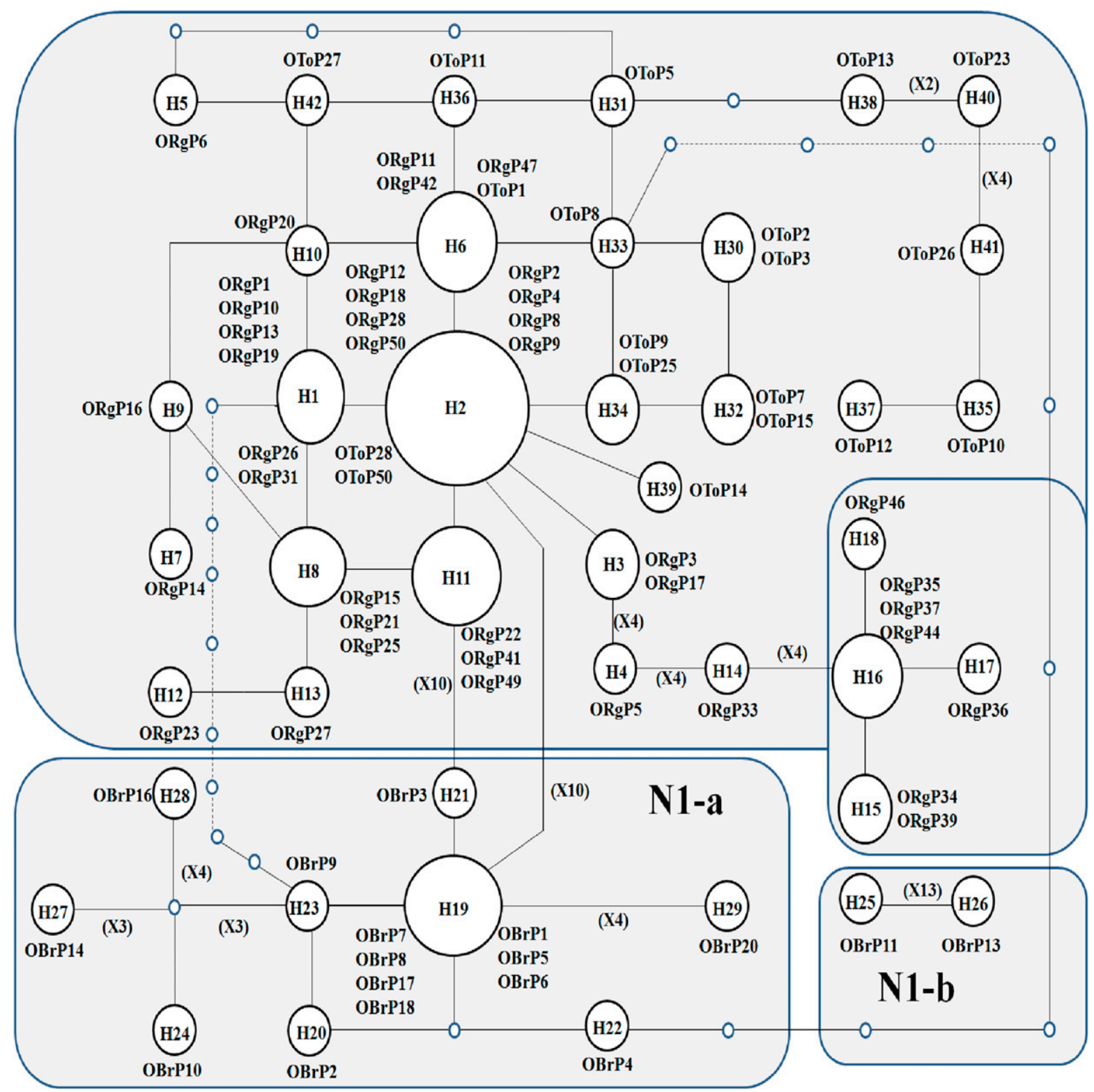

Fig. 4. Network based on the combined sequences of the three mitochondrial regions (rpl5-rns, rns-cox2, and cox2+spacer) of the 76 Phytophthora nicotianae isolates analyzed in the current study. The network was constructed using a statistical parsimony algorithm implemented in TCS 1.21 (Excoffier et al. 2005). 
variability, respectively. The percentage of variation was higher among the populations when compared with within populations both in the nuclear (67.70) and mitochondrial regions (53.99). A high fixation index $\left(\mathrm{F}_{\mathrm{ST}}\right)$ value $(0.67698)$ was obtained from the AMOVA of nuclear genotypes but significant values were also obtained with mitochondrial haplotypes (0.53990).

\section{DISCUSSION}

A combined analysis of phenotypic, molecular, and pathogenicity data has been applied in the current study to characterize $P$. nicotianae isolates responsible for severe outbreaks of fruit rots in brinjal, ridge gourd, and tomato in South India. This study assumed significance in understanding whether the population responsible for such severe outbreaks evolved through genetic recombination or migrated from elsewhere and is crucial for developing effective disease management strategies and also for the selection of the appropriate pathogen isolates to use in a resistance screening program. The results of this study showed that different genotypes of $P$. nicotianae are responsible for severe outbreaks of fruit rots in brinjal, ridge gourd, and tomato in South India. This is the first report of the involvement of $P$. nicotianae causing severe outbreaks of fruit rot in ridge gourd and brinjal in India, even though it was recorded earlier on tomato as rare incidences. The morphological parameters of isolates of $P$. nicotianae were in agreement with available literature (Hall 1993; Ho and Jong 1989; Stamps et al. 1990; Tucker 1931; Waterhouse 1963) and did not differentiate characterized isolates. In the present study, the population structure and the host-specific genetic relationships among $P$. nicotianae isolates was determined using three mitochondrial and three nuclear regions from 76 isolates collected from brinjal, tomato and ridge gourd. Network analyses of combined nuclear markers revealed a significant grouping of isolates in relation to the host. Haplotype diversity (0.816) was higher in the $\beta$-tub gene compared with other nuclear regions. This was due to the presence of a higher number of heterozygous SNPs in the $\beta$-tub gene. Host-specific grouping of brinjal isolates were observed in the networking of both nuclear and mitochondrial sequences. Pairwise $\mathrm{F}_{\mathrm{ST}}$ comparisons obtained from the AMOVA of nuclear regions (hyp, scp, and $\beta$-tub) (0.67698) revealed variation among $P$. nicotianae isolates. Previous studies have also shown that nucleotide diversity is higher in nuclear loci than mitochondrial loci for P. capsici (Quesada-Ocampo et al. 2011) and $P$. parasitica (Forster et al. 1990), which is similar to findings in our present study. A previous study based on combined mitochondrial and nuclear markers in P. nicotianae found citrus isolates from Italy, California, Florida, Syria, Albania, and the Philippines clustered together in the same clade and isolates recovered from Nicotiana and Solanum spp. were grouped as separate clusters (Mammella et al. 2013). A specific population structuring based on nuclear RFLPs has also been reported for isolates from tobacco that was differentiated from other P. nicotianae isolates (Colas et al. 1997). However, these studies could not conduct specific pathogenicity tests to confirm whether molecular groupings matched physiological race or pathotype profiles.

In the present study, all isolates of $P$. nicotianae were significantly more aggressive on the original host but were also able to cause infection on alternative hosts. Considerable evidence is available to show that some isolates of $P$. nicotianae are host-specific. For example, a $P$. nicotianae isolate from okra was not pathogenic to Citrus spp. and vice versa (Erwin 1964). Similarly, isolates from Citrus spp. were more virulent on rough lemon than isolates from petunia, tomato, walnut, silk tree, jojoba, hibiscus, and peach

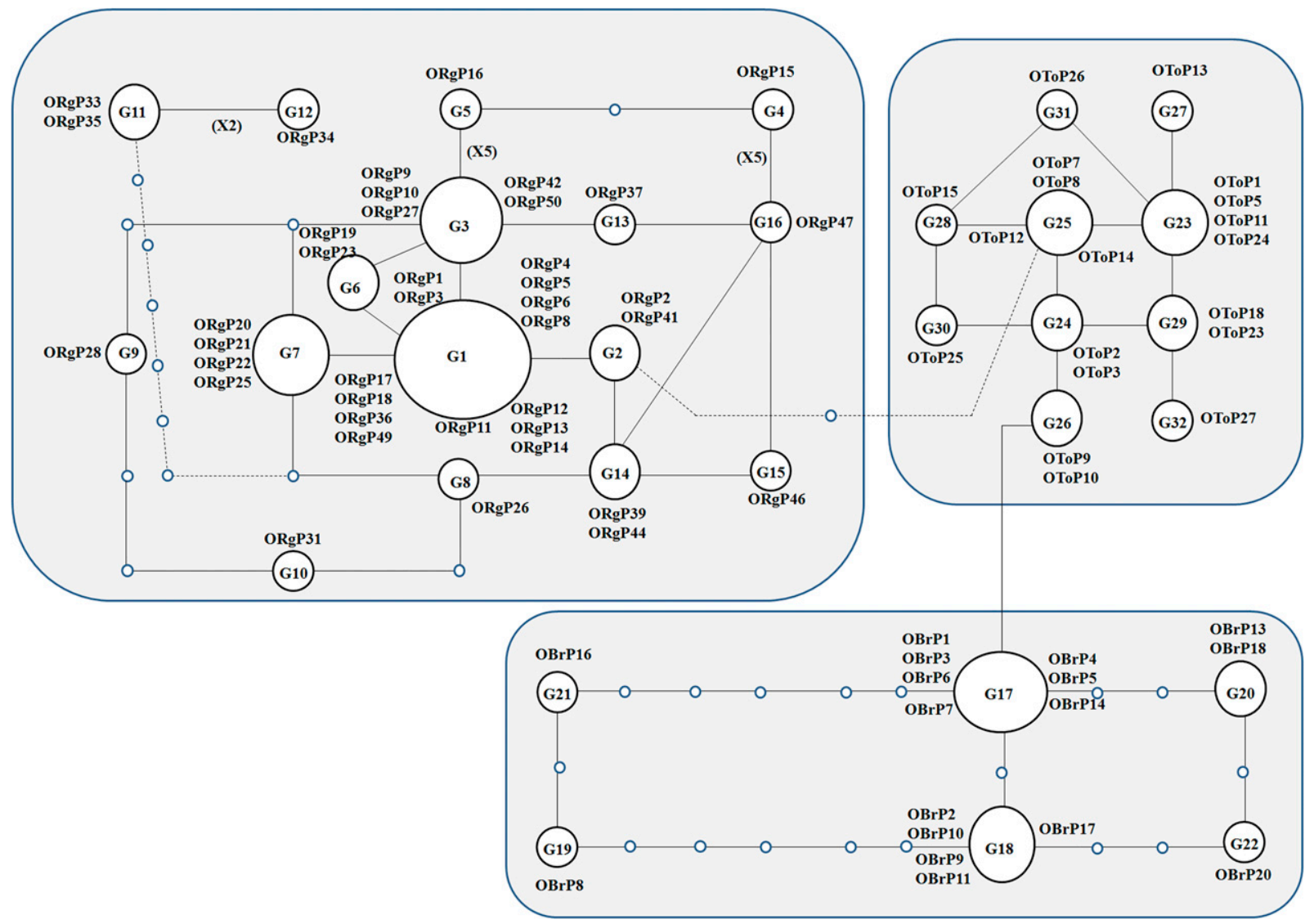

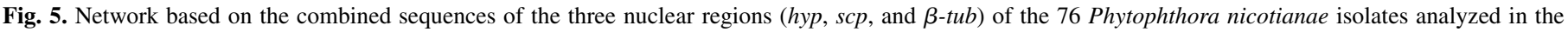

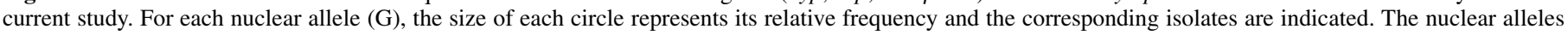

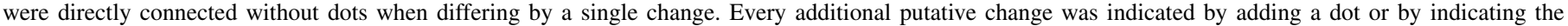
number of steps on dotted lines. 
(Matheron and Matejka 1990). In another study, tomato plants exhibited high susceptibility to many isolates, including Citrus isolates (Bonnet et al. 1978). These studies indicated that the degree of susceptibility among hosts is not clear but the greatest degree of virulence is commonly shown by isolates on their own host. A similar kind of differential host adaptation of $P$. infestans to potato and tomato has been reported in Ecuador (Oyarzun et al. 1998) and Uganda and Kenya (Vega-Sanchez et al. 2000). Although buckeye rot was known on tomato in India since 1961 (Jain et al. 1961), fruit rots on brinjal and ridge gourd were not reported until 2011 and 2013, respectively. The population attacking brinjal and ridge gourd may have been introduced from another location where it evolved independently of tomato, as outbreaks of fruit rot on brinjal and on ridge gourd were recorded in 2011 and 2013, respectively.

The existence of significant structuring in relation to host could suggest phenomena of migration of the isolates through plant material or host adaptation (Mammella et al. 2013). In the present study, the pathogenicity tests on different hosts suggest that molecular groupings identified can be taken as evidence of physiological races or pathotypes. Based on the results obtained in this study it can be hypothesized that $P$. nicotianae isolates might have migrated from other areas with infected plant material or that lineages may have progressively diverged and caused severe outbreaks on ridge gourd and brinjal, as fruit rots were not reported on these crops prior to 2011. The role of the global nursery trade in the spread of $P$. nicotianae has been investigated and found that isolates from Dieffenbachia maculata from Germany and Florida shared the same mitochondrial haplotype and nuclear multilocus genotype (Mammella et al. 2013). During recent surveys, P. nicotianae has been widely recovered in nurseries and its long-distance movement can likely be facilitated by its broad host range, representing more than 250 plant genera in 90 families, including a large number of ornamentals (Hulvey et al. 2010; Moralejo et al. 2009; Pane et al. 2005; Schwingle et al. 2007; Yakabe et al. 2009).

Metalaxyl and its isomer mefenoxam have been used to manage plant diseases caused by the oomycete pathogens in various crops including herbaceous annuals (Cohen and Coffey 1986; Erwin and Ribiero 1996). Previous studies have shown that metalaxyl/ mefenoxam-resistance in $P$. nicotianae occurred predominantly on herbaceous annuals, but rarely on perennials (Hu et al. 2008; Hwang and Benson 2005). In the present study, all the isolates of $P$. nicotianae were sensitive to metalaxyl, which may be due to lack of exposure of brinjal, ridge gourd, and tomato crops in South India to the metalaxyl until 2012 as severe outbreaks of Phytophthora diseases were not recorded on these crops. The continuous use of metalaxyl increases the probabilities for development of resistance to it. $P$. nicotianae is largely a foliar pathogen on these annuals and disease incidences on those crops are usually high and several applications of metalaxyl/mefenoxam are required to manage these foliar diseases. The repeated exposure of $P$. nicotianae to these fungicides may lead to faster resistance development due to selection pressure. Even though all isolates were sensitive to metalaxyl in our study, it is warranted not to recommend metalaxyl for management of fruit rot on vegetable crops in India.

In conclusion, a combined analysis of nuclear DNA sequences revealed host-specific populations in $P$. nicotianae that were responsible for severe outbreaks of fruit rots in brinjal, ridge gourd, and tomato in India. This is the first report of differential host adoption of $P$. nicotianae to brinjal, tomato, and ridge gourd, and indicates preferential reproduction systems and the role of planting material in the distribution of the species. It is not clear whether host-specific populations have migrated or evolved in India through sexual recombination or any other mechanism. Furthermore, $P$. nicotianae has not been reported on ridge gourd in any part of the world. Whole genome sequence analysis of hostspecific groups and studies of the pathogen population structure over time will provide information on its evolutionary history that can help in the effective deployment of host resistance and fungicides for disease management. From this study, it is not possible to understand the extent of spread of host-specific groupings of $P$. nicotianae. A large-scale survey and population analysis of Phytophthora across brinjal, ridge gourd, and tomato growing regions of India is warranted. Genotyping of $P$. nicotianae isolates from tobacco and citrus is necessary to understand relationships with isolates from brinjal, tomato, and ridge gourd and to track the diversification of inferred host-specific clades and to identify new ones. Finally, this study of the genetic structure of $P$. nicotianae populations can be used as a reference guide for the selection isolates to enhance the efficacy of breeding programs.

\section{ACKNOWLEDGMENTS}

We are highly thankful to the Indian Council of Agricultural Research, New Delhi for financial support in the form of ALCOCERA, an outreach programme on Alternaria, Colletotrichum, and Cercospora diseases of field and horticultural crops.

\section{LITERATURE CITED}

Álvarez, L. A., Pérez-Sierra, A., Armengol, J., and García-Jiménez, J. 2007. Characterization of Phytophthora nicotianae isolates causing collar and root rot of lavender and rosemary in Spain. J. Plant Pathol. 89:261-264.

Blair, J. E., Coffey, M. D., Park, S. Y., Geiser, D. M., and Kang, S. 2008. A multi-locus phylogeny for Phytophthora utilizing markers derived from complete genome sequences. Fungal Genet. Biol. 45:266-277.

Bonnet, P., Maia, N., Tello-Marquina, J., and Venard, P. 1978. Pouvoir pathogène du Phytophthora parasitica (Dastur): Facteurs de variabilité et notion de spécialisation parasitaire. Ann. Phytopathol. 10:15-29.

Cacciola, S. O., and Magnano di San Lio, G. 2008. Management of citrus diseases caused by Phytophthora spp. Pages 61-84 in: Integrated Management of Diseases Caused by Fungi, Phytoplasma and Bacteria. A. Ciancio and K. G. Mukerji, eds. Springer Science+Business Media B.V., Dordrecht, The Netherlands.

Chowdappa, P., Brayford, D., Smith, J., and Flood, J. J. 2003. Molecular discrimination of Phytophthora isolates on cocoa and their relationship with coconut, black pepper and bell pepper isolates based on rDNA repeat and AFLP fingerprints. Curr. Sci. 84:1235-1238.

Clement, M., Posada, D., and Crandall, K. A. 2000. TCS: A computer program to estimate gene genealogies. Mol. Ecol. 9:1657-1659.

Cohen, Y., and Coffey, M. D. 1986. Systemic fungicides and the control of oomycetes. Annu. Rev. Phytopathol. 24:311-338.

Colas, V., Lacourt, I., Ricci, P., Vanlerberghe-Masutti, F., Venard, P., Poupet, A., and Panabières, F. 1998. Diversity of virulence in Phytophthora parasitica on tobacco, as reflected by nuclear RFLPs. Phytopathology 88: 205-212.

Erwin, D. C. 1964. A strain of Phytophthora parasitica from okra and its sexual compatibility with isolates from citrus. Phytopathology 54:114-115.

Erwin, D. C., and Ribiero, O. K. 1996. Phytophthora Diseases Worldwide. The American Phytopathological Society, St. Paul, MN.

Excoffier, L., Laval, G., and Schneider, S. 2005. Arlequin ver. 3.0: An integrated software package for population genetics data analysis. Evol. Bioinform. Online 1:47-50.

Forbes, G. A., Escobar, X. C., Ayala, C. C., Revelo, J., Ordonez, M. E., Fry, B. A., Doucett, K., and Fry, W. E. 1997. Population genetic structure of Phytophthora infestans in Ecuador. Phytopathology 87:375-380.

Forster, H., Oudemans, P., and Coffey, M. D. 1990. Mitochondrial and nuclear DNA diversity within six species of Phytophthora. Exp. Mycol. 14:18-31.

Grünwald, N. J., and Goss, E. M. 2011. Evolution and population genetics of exotic and re-emerging pathogens: Novel tools and approaches. Annu. Rev. Phytopathol. 49:249-267.

Hall, G. 1993. An integrated approach to the analysis of variation in Phytophthora nicotianae and a re-description of the species. Mycol. Res. 97: $559-574$.

Hall, T. A. 1999. BioEdit: A user-friendly biological sequence alignment editor and analysis program for Windows 95/98/NT. Nucleic Acids Symp. Ser. 41:95-98.

Ho, H. H., and Jong, S. C. 1989. Phytophthora nicotianae (P. parasitica). Mycotaxon 35:243-276.

Hu, J. H., Hong, C. X., Stromberg, E. L., and Moorman, G. W. 2008. Mefenoxam sensitivity and fitness analysis of Phytophthora nicotianae isolates from nurseries in Virginia, USA. Plant Pathol. 57:728-736.

Huelsenbeck, J. P., and Ronquist, F. 2001. MrBayes: Bayesian inference of phylogenetic trees. Bioinformatics 17:754-755. 
Hulvey, J., Gobena, D., Finley, L., and Lamour, K. 2010. Co-occurrence and genotypic distribution of Phytophthora species recovered from watersheds and plant nurseries of eastern Tennessee. Mycologia 102:1127-1133.

Hwang, J., and Benson, D. M. 2005. Identification, mefenoxam sensitivity, and compatibility type of Phytophthora spp. attacking floriculture crops in North Carolina. Plant Dis. 89:185-190.

Jain, S. S., Sharma, S. L., and Juneja, S. L. 1961. Studies on buck eye rot of tomato-new to Himachal Pradesh. Proc. Indi. Sci. Cong. Assoc. 50:351-352.

Johnson, E. S., Wolff, M. F., Wernsman, E. A., Atchely, W. R., and Shew, H. D. 2002. Origin of the black shank resistance gene, $\mathrm{Ph}$, in tobacco cultivar Coker 371-gold. Plant Dis. 86:1080-1084.

Kong, P., Hong, C. X., and Richardson, P. A. 2003. Rapid detection of Phytophthora cinnamomi using PCR with primers derived from the Lpv putative storage protein genes. Plant Pathol. 52:681-693.

Lamour, K. H., Daughtrey, M. L., Benson, D. M., Hwang, J., and Hausbeck, M. K. 2003. Etiology of Phytophthora drechsleri and P. nicotianae $(=P$. parasitica $)$ diseases affecting floriculture crops. Plant Dis. 87:854-858.

Librado, P., and Rozas, J. 2009. DnaSP v5: A software for comprehensive analysis of DNA polymorphism data. Bioinformatics 25:1451-1452.

Mammella, M. A., Cacciola, S. O., Martin, F., and Schena, L. 2011. Genetic characterization of Phytophthora nicotianae by the analysis of polymorphic regions of the mitochondrial DNA. Fungal Biol. 115:432-442.

Mammella, M. A., Martin, F. N., Cacciola, S. O., Coffey, M. D., Faedda, R., and Schena, L. 2013. Analyses of the population structure in a global collection of Phytophthora nicotianae isolates inferred from mitochondrial and nuclear DNA sequences. Phytopathology 103:610-622.

Martin, F. N., and Tooley, P. W. 2003. Phylogenetic relationships among Phytophthora species inferred from sequence analysis of mitochondrially encoded cytochrome oxidase I and II genes. Mycologia 95:269-284.

Matheron, M. E., and Matejka, J. C. 1990. Differential virulence of Phytophthora parasitica recovered from Citrus and other plants to rough lemon and tomato. Plant Dis. 74:138-140.

Moralejo, E., Pérez-Sierra, A. M., Álvarez, L. A., Belbahri, L., Lefort, F., and Descals, E. 2009. Multiple alien Phytophthora taxa discovered on diseased ornamental plants in Spain. Plant Pathol. 58:100-110.

Oyarzun, P. J., Pozo, A., Ordonez, M. E., Doucett, K., and Forbes, G. A. 1998. Host specificity of Phytophthora infestans on tomato and potato in Ecuador. Phytopathology 88:265-271.

Pane, A., Martini, P., Chimento, A., Rapetti, S., Savona, S., Grasso, F. M., and Cacciola, S. O. 2005. Phytophthora species on ornamental plants in Italy. J. Plant Pathol. S87:301.

Parkunan, V., Johnson, C. S., Bowman, B. C., and Hong, C. X. 2010. Population structure, mating type, and mefenoxam sensitivity of Phytophthora nicotianae in Virginia tobacco fields. Plant Dis. 94:1361-1365.

Prigigallo, M. I., Abdelfattah, A., Cacciola, S. O., Faedda, R., Sanzani, S. M., Cooke, D. E. L., and Schena, L. 2015a. Metabarcoding analysis of Phytophthora diversity using genus-specific primers and 454 pyrosequencing. Phytopathology 3:305-313.

Prigigallo, M. I., Mosca, S., Cacciola, S. O., Cooke, D. E. L., and Schena, L. 2015b. Molecular analysis of Phytophthora diversity in nursery-grown ornamental and fruit plants. Plant Pathol. 64:1308-1319.

Quesada-Ocampo, L. M., Granke, L. L., Mercier, M. R., Olsen, J., and Hausbeck, M. K. 2011. Investigating the genetic structure of Phytophthora capsici populations. Phytopathology 101:1061-1073.

Raeder, U., and Broda, P. 1985. Rapid preparation of DNA from filamentous fungi. Appl. Microbiol. 1:17-20.

Ronquist, F., and Huelsenbeck, J. P. 2003. MrBayes 3: Bayesian phylogenetic inference under mixed models. Bioinformatics 19:1572-1574.

Schena, L., Cardle, L., and Cooke, D. 2008. Use of genome sequence data in the design and testing of SSR markers for Phytophthora species. BMC Genomics 9:620.

Schena, L., and Cooke, D. E. L. 2006. Assessing the potential of regions of the nuclear and mitochondrial genome to develop a "molecular tool box" for the detection and characterization of Phytophthora species. J. Microbiol. Methods 67:70-85.

Schwingle, B. W., Smith, J. A., and Blanchette, R. A. 2007. Phytophthora species associated with diseased woody ornamentals in Minnesota nurseries. Plant Dis. 91:97-102.

Stamps, D. J., Waterhouse, G. M., Newhook, F. J., and Hall, G. S. 1990. Revised tabular key to the species of Phytophthora. Mycol. Pap. No. 162. International Mycological Institute, Kew, United Kingdom.

Sullivan, M. J., Parks, E. J., Cubeta, M. A., Gallup, C. A., Melton, T. A., Moyer, J. W., and Shew, H. D. 2010. An assessment of the genetic diversity in a field population of Phytophthora nicotianae with a changing race structure. Plant Dis. 94:455-460.

Tucker, C. M. 1931. Taxonomy of the genus Phytophthora de Bary. University of Missouri College of Agriculture, Agricultural Experiment Station. Res. Bull. (Sun Chiwawitthaya Thang Thale Phuket) 153:168-176.

Vega-Sanchez, M. E., Erselius, L. J., and Rodriguez, A. M. 2000. Host adaptation to potato and tomato within the US-1 clonal lineage of Phytophthora infestans in Uganda and Kenya. Plant Pathol. 49:531-539.

Waterhouse, G. M. 1963. Key to the species of Phytophthora de Bary. Mycol. Pap. No. 92:1-22. International Mycological Institute, Kew, United Kingdom.

Yakabe, L. E., Blomquist, C. L., Thomas, S. L., and MacDonald, J. D. 2009. Identification and frequency of Phytophthora species associated with foliar diseases in California ornamental nurseries. Plant Dis. 93: 883-890.

Zhang, X. G., Sun, W. X., Guo, L., Yu, J. F., and Chang, C. J. 2003. Genetic and pathogenic variation among tobacco black shank strains of Phytophthora parasitica var. nicotianae from the main tobacco growing in China. J. Phytopathol. 151:259-266. 